\title{
Recuperación del patrimonio histórico-artístico de San Marcos
}

\author{
Ricardo Estabridis Cárdenas* \\ Departamento Académico de Arte
}

El patrimonio histórico artístico mueble de la Universidad de San Marcos constituído por obras que proceden de años coloniales, republicanos y contemporáneos se ha reunido en el hoy llamado Museo de Arte, donde se integran todas sus colecciones. Entre ellas una de las más importantes, es la de retratos de personajes ilustres de la Universidad, obras que nos presentan a catedráticos, decanos y rectores que a lo largo de los siglos, desde su fundación, gestaron el prestigio de esta Casa de Estudios, Decana de América.

La colección de retratos del Museo de Arte la conforman 143 lienzos que, aparte de su importancia como documento histórico, reflejan en conjunto la evolución de los estilos pictóricos en la historia del retrato peruano.

Desde que se fundố éste Museo, hace 27 años, sólo se han exhibido en sus salas de exposiciones permanentes 29 retratos, los demás permanecieron en los depósitos por su mal estado de conservación. Hoy gracias a gestiones iniciadas años atrás ante la Agencia Española de Cooperación Internacional, en base al Convenio Perú- España, y después de un proceso que duró aproximadamente dos años, se restauraron dieciseis lienzos de singular calidad plástica.

En la exposición inaugurada con motivo de lacelebración del $446^{\circ}$ aniversario de nuestra Universidad, podemos ver el fruto de estos esfuerzos en la brillante tarea realizada por los restauradores, equipo dirigido en una primera etapa por la señora Esperanza García y actualmente por el señor Enrique Castilla. Ellos han recuperado 16 lienzos de los siglos XVII, XVIII, XIX y XX que se estaban perdiendo para la historia de San Marcos y para la historia del arte de nuestro país.

* Director del Museo de Arte de la Universidad Nacional Mayor de San Marcos.

LETRAS (Lima), 94: 25-36,1997. 
El presente ensayo pretende trazar un perfil del desarrollo del arte del retrato en Lima en base a las obras restauradas, ya que las consideramos representativas de los siglos en que se pintaron. Además paralelamente destacaremos la importancia del personaje retratado para la historia de la Universidad ${ }^{1}$.

\section{Los retratos de personajes del siglo XVI}

Existen en la colección del Museo de Arte de San Marcos varios lienzos que perennizaron para la posteridad la imagen de los personajes que sembraron la semilla del saber en los claustros sanmarquinos en los primeros lustros de su existencia. Entre ellos destacamos los retratos de Gerónimo López Guarnido y Luis López de Solís, obras consideradas de los pintores italianos Bernardo Bitti y Mateo Perez de Alesio respectivamente ${ }^{2}$. A dichas obras se pueden hoy sumar en importancia los lienzos restaurados de Domingo de Santo Tomás, Antonio Hervias y Juan de Lorenzana, que están bajo la égida de la influencia de la pintura italiana.

Entre 1575 y 1625 , aproximadamente, se hicieron presentes en nuestras tierras tres pintores italianos que dejaron una profunda huella en la pintura peruana a través de su obra y de su magisterio: el hermano jesuita Bernardo Bitti, Matęo Péreżé Alesio y AngelínodMedoro ${ }^{3}$. El primero en llegar a Lima fue Bitti, considerado elmejospintor de, Sudamérica del siglo XVI, del cual sólo se conocía obra religiosa hasta que el doctor Francisco Stastny identificara el retrato de Gerónimo López Guarnido. El segundo fue Alesio, precedido de una brillante trayectoria que nos lleva hasta la Capilla Sixtina, donde dejara a la posteridad una de sus pinturas; ya en el Perú, según atribución de Bruno Roselli se cuenta entre sus obras profanas, el retrato de Luis López de Solís.

La escuela de Alesio integrada por un buen número de discípulos

1 Los únicos escritos que existen sobre la colección desde el punto de vista históricoartístico son los de Francisco Stastny: La Pintura Limeña y los Retratos de la Universidad de San Marcos, Lima, 1975, catálogo editado por la Universidad, y el de Ricardo Estabridis: Cuatro Siglos del Retrato en la Historia de Lima: Pinacoteca de San Marcos,. Lima, 1994, catálogo editado por el Banco Continental.

2 Stastny, op.cit, pp. 12 y 14.

3 Estabridis, Ricardo: "Influencia Italiana en la Pintura Virreinal". En Pintura en el Virreinato del Perú, editado por el Banco de Crédito del Perú . Lima, 1989, p. 109. 
acaparó los encargos de la época sobre todo en el tránsito hacia el siglo XVII, buen ejemplo de ello son los retratos póstumos del fundador de la Universidad, así como los de Domingo de Santo Tomás y Antonio Hervias, que presentan caracteres estilísticos que los hermanan entre sí, tales como la despreocupación por el realismo en el tratamiento de las vestiduras, cierta idealización en los rostros y el gusto por los fondos neutros sin cortinajes.

En estos lienzos se perpetuó la imagen de dos frailes dominicos que tuvieron que ver con el comienzo y fin de la regencia dominica en la Universidad de San Marcos: Fray Domingo de Santo Tomás y Antonio Hervias.

Fray Domingo de Santo Tomás, nacido en Sevilla al promediar el siglo $\mathrm{XV}$, fue uno de los siete primeros frailes dominicos que llegaron al Perú en el grupo que acompañó a Pizarro en la expedición conquistadora ${ }^{4}$. Años después colaboró con Fray Tomás de San Martín en la organización inicial de la Universidad donde se desempeñaria como Primer Catedrático de Prima de Teología y Regente Mayor de Estudios. Llegó a ser Prior del Convento del Rosario de Lima, así como Visitador y Vicario General de la Provincia. En tiempos del Arzobispo Jerónimode Loayza se le consagró como Obispo de La Plata donde murió en 1570 . Es considerado uno de los primeros quechuistas ya que estudió esta lengua y publicó: El Arte de la lengua General Quechua 5 .

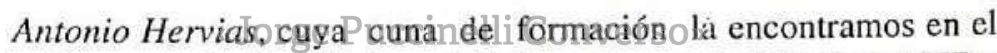
afamado Convento de San Esteban de Salamanca, pasó al Perú en 1557 y formó parte del claustro de la Universidad con la cátedra de Prima de Sagrada Teología y posteriormente como rector entre $1565-66$ y por segunda vez en 1571. En este último año la Universidad que sólo obstentaba el título de Real se convirtió por bula del Papa Pío V en la primera universidad de América con 4 rango de Real y Pontificia ${ }^{6}$. Antonio Hervias fue el último rector dominico, ya que hasta 1571 fueron sólo los priores de esa Orden los que asumían dichos cargos. Sin embargo sus dignidades se acrecentaron cuando fue nombrado Obispo de Arequipa, y luego de Verapaz y Cartagena de Indias, donde murió en $1590^{7}$.

4 Eguiguren, Luis Antonio: Diccionario Histórico Cronológico. T. I, pp. 825-827. Lima, 1940.

5 Eguiguren, Luis A.: Alma Mater. pp. 118-119. Lima, 1939.

6 Valcárcel, Daniel: Recibimientos a San Martín y Bolívar en la Universidad de San Marcos. p. 23. Lima, 1951.

7 Eguiguren, L.A.: La Universidad en el siglo XVI. T. I, p. 351. Lima, 1951. Diccionario...., p. 821. 
Fray Juan de Lorenzana, al igual que Antonio Hervias, provenía del Convento de San Esteban de Salamanca y pasó al Perú en 1590 donde llegó a ser Prior Provincial de los dominicos y Calificador del Santo Oficio. En 1606 fundó la Recolección de Santa María Magdalena, que se ubicaba en la hoy llamada Plaza de la Recoleta. En San Marcos tuvo a su cargo la cátedra de Prima de Sagrada Teología. Su memoria está ligada a la vida de Santa Rosa de Lima, de quien fuera su confesor y guía espiritual ${ }^{8}$.

El retrato de Lorenzana por sus caracteres formales, creemos que está dentro de. la escuela de Angelino Medoro, además por noticias históricas sabemos que Lorenzana y Medoro pertenecieron al círculo de Santa Rosa, en la casa de don Gonzalo de la Maza. Este retrato fue restaurado gracias al apoyo del Banco de Crédito del Perú para integrar la exposición que se llevó a cabo en la Catedral de Lima, dentro del marco del homenaje que le hiciera a la Santa limeña esta institución bancaria en 1995, e incluído en el libro: "Santa Rosa de Lima y su Tiempo"?.

Contemporáneo de Lorenzana fue Juan Bautista Villalobos quien tuvo a su cargo la cátedra de Vísperas de Sagrados Canones, creada en tiempos del rector Marcos de Lucio, Su retrato reune caracteres similares a los anotados para el lienzo anterior.

\section{Los retratos de persoñajes del sigla XVu Letras \\ "Jorge Puccinelli Converso"}

En la pintura limeña de las primeras décadas del siglo XVII, la escuela de la trilogía de pintores italianos citados líneas atrás, se prolonga en la obra de los llamados manieristas tardíos, quienes además reciben la influencia del naturalismo de los manieristas de Amberes a través de los grabados y del claroscurismo de la pintura española enviada de la metrópoli.

En el círculo de los pintores de esta época destaca Antonio Mermejo, del cual el Museo posee el retrato de Juan de la Reinaga Salazar. Por sus caracteres formales, incluimos también en esta línea estilística el lienzo restaurado con el retrato de don Alvaro de Ibarra. En los artistas de esta época ya se aprecia una primera preocupación por ciertos caracteres naturalistas en base al claroscuro, que anuncian la tendencia hacia el estilo barroco ${ }^{10}$.

\footnotetext{
8 Eguiguren, L.A.: Op. cit, T. I, pp. 239 y 853.

9 Varios: Santa Rosa de Lima y su tiempo. Editado por el Banco de Crédito del Pení. Lima, 1995. p. 114.

10 Stastny, Francisco: "Jaramillo y Mermejo caravaggistas limeños". En Cielo Abierto. Vol. IX, № 27. Enero-Marzo, 1984.
} 
Alvaro de Ibarra, fue un ilustre limeño nacido en 1621, de destacada trayectoria en los años que le tocó vivir. Apenas con venticinco años ganó las oposiciones en la Universidad de San Marcos para la cátedra de Prima de Leyes. En la vida universitaria tuvo activa participación en las reformas que se dieron en tiempos del Virrey Conde de Castellar sobre las cátedras vacantes, innovaciones que incluso se adoptaron más adelante en las universidades de Salamanca, Valladolid y Alcalá ${ }^{11}$. Fue Inquisidor Apostólico de Lima y después Regente Mayor y Visitador de esta Real Audiencia, e incluso fue nombrado Obispo de Trujillo sin ser sacerdote ${ }^{12}$.

En el desarrollo de la pintura limeña del siglo XVII, al incipiente naturalismo de Mermejo sigue ya el dominio pleno del realismo, tal como sucedió en la pintura barroca española. En nuestra colección ello se pone de manifiesto en el retrato de don Diego de Vergara, y en especial en el retrato de Cipriano de Medina recientemente restaurado, trabajo que ha permitido recuperar uno de las mejores obras del patrimonio pictórico de San Marcos, donde se consigue a plenitud el realismo en la gran fuerza expresiva de los caracteres fisonómicos del representado.

Fray Cipriano de Medina, nació en Lima, fue hijo del doctor Cipriano de Medina, abogado de la Real Audiencia de Lima y en dos oportunidades Rector de San Marcos, Yjdedoña Sebastiana de Vegă y Padilla, hermana de don Feliciano de la Vega y Padillae quiencasímismo fuera rector de San Marcos en tres períodos.

La vida religiosa de Cipriano de Medina está ligada a la de San Martín de Porres, quien influyó para que ingresara al Convento del Rosario de Lima. Eguiguren nos relata otro episodio de su vida, que corresponde a un milagro del santo moreno, por el cual después de los cuidados que le dió en una enfermedad de varios meses, hizo que transformara su físico, levantando al enfermo con media vara más de estatura y mejor semblante, evitando con ello que continuara la mofa de que había sido objeto por sus compañeros del noviciado ${ }^{13}$.

11 Mendiburu, Manuel de: Diccionario histórico-biográfico del Perú, T. VI, pp. 327-328. Lima, 1933.

12 Tauro, Alberto: Diccionario enciclopédico del Perú, T. II, pp. 142-143. Buenos Aires, 1966.

13 Eguiguren, L.A.: Op cit., T. I, pp. 544-545 y 822-823. Lima, 1940. 
En la Universidad de San Marcos fue el primer catedrático de Teología Moral, cátedra creada por el rey Felipe IV para que sea leída por un dominico nacido en el Perú y en la cual se estudiase la doctrina de Santo Tomás ${ }^{14}$. Llegó a ser Obispo de Huamanga y Calificador del Tribunal de la Inquisición. Murió en Huamanga en 1664.

Un retrato de tránsito entre el siglo XVII y XVIII es el de Diego Montero del Aguila, vestido a la moda de Carlos II, con la austeridad propia de los augsburgo, acompañado por su escudo nobiliario, con fondo de biblioteca y mesa con dignidades episcopales. Según Eguiguren el nieto de este personaje, José Morales de Aramburú y Montero colocó este retrato en la Capilla de la Universidad en $1769^{15}$.

Diego Montero del Aguila, nació en Santiago de Chile, hijo del matrimonio de don Diego Gonzales Montero y Justiniano, quien fuera dos veces Presidente del Reino de Chile, y de doña Ana Sarmiento del Aguila. Estudió en el Colegio Real de San Martín y por su sabiduría en Leyes fue hombre de confianza del Santo Tribunal de la Inquisición. Su vida cambió al enviudar en 1696 ya que ingresó a la vida religiosa. En 1698 fue nombrado Cura Rector de la Catedral de Lima y en 1707 Provisor Vicario General y Gobernador de este Arzobispado. En la Universidad de San Marcos desde 1687 hasta 1708 tuvo a su cargo la eâtedrade Prima de Leyes. En 1709 fue nombrado por Felipe V Obispo de la Concepción de Chile y años después, en 1716, fue promovido al obispado de Trujillo. Murió en Saña dos años más tarde ${ }^{16}$.

\section{Los retratos de personajes del siglo XVIII}

En el siglo XVIII la escuela limeña se desarrolla plenamente como fruto de influencias italianas, españolas y flamencas recibidas en los siglos anteriores, con caracteres que demuestran el dominio en las técnicas para conseguir el realismo en el género retratístico. Clara muestra de ello es el retrato del franciscano Gregorio Martínez Ronceros, donde además es posible apreciar el gusto por agregar como marco del representado los fondos de biblioteca, escudos y cartelas con leyendas, en un afán de indicar su erudición y justificar ante nosotros el porqué de su transcendencia histórica.

14 Valcárcel, Daniel: Historia de la Educación Colonial, p. 185.

15 Eguiguren, L.A.: Op. cit., T. I, p. 549.

16 Eguiguren, L.A.: Op. cit., T. I, pp. 824-825. 
Gregorio Martínez Ronceros, nacido en la Villa de Pisco, perteneció a la orden franciscana, de la que llegó a ser su Ministro Provincial. En 1703 cuando gobernaba el Virrey Conde de la Moncloa se instaló en San Marcos la cátedra de Prima de Scoto, concedida por su majestad el Rey Felipe V mediante una real cédula expedida el 9 de abril de 1701, a favor de la religión seráfica. Gregorio Martínez Ronceros fue nombrado su primer catedrático ${ }^{17}$.

En 1700 el cambio de dinastía en España de los Augsburgo por los Borbones, con la coronación de Felipe V, va a significar para España y sus colonias un giro no sólo en el campo del pensamiento sino también en la forma de vida, y por consecuencia en las formas artísticas. En estos años en la escuela limeña prolifera el retrato de gala, donde los personajes son representados con vestidos a la moda francesa y pelucas empolvadas. Un buen ejemplo de ello lo apreciamos en el retrato del que fuera rector de San Marcos entre 1740-42, don Diego Hurtado de Mendoza.

Diego Hurtado de Mendoza, hijo de Diego Hurtado de Mendoza y Jaraquemada y de Catalina Angela de Iturrizarra, nació en Lima en 1694, donde realizó estudios en el Real Colegio de San Martín y posteriormente en San Felipe y San Marcos, graduándose de Doctor en Leyes y Cánones. Su profesión y cualidades lo llevaron a ser abogado de la Real Audiencia y de Presos del Santo Oficio y a las cátedras sanmarquinas. Fue elegido Rector de San Marcos en 1740 ymurió en ejercicio de su cargo en $1742^{18}$.

En el caso de los retratos de religiosos no aparecen las pelucas empolvadas, sin embargo la moda se pone de manifiesto en el gusto por el detalle ornamental en el fino trabajo de los encajes y bordados del traje eclesiástico, como es posible apreciar en el retrato del mercedario limeño Francisco Gutierrez Galeano, catedrático sanmarquino en la primera mitad del siglo XVIII.

En la colección de retratos diciochescos deseamos destacar los lienzos que perennizaron para la posteridad las figuras de tres religiosos mercedarios en la primera mitad del siglo: Francisco Gutierrez Galeano, José de Castro y Feliciano Palomares.

17 Eguiguren, L.A.: Catálogo Histórico del Claustro de la Universidad de San Marcos. (1756-1800), p. 73.

18 Tauro, Alberto: Enciclopedia Ilustrada del Perú, T. 3, p. 1027. Lima,1987. 
Francisco Gutierrez Galeano, nació en Lima en 1670, hijo de Francisco Gutierrez Galeano y de Leonarda García Rangel. Ingresó a la orden de la Merced, donde llegó a ser Rector del Colegio de San Pedro Nolasco y Provincial de la orden en 1727. Entre sus dignidades eclesiásticas se cuentan la de Calificador, Consultor y Juez Ordinario del Santo Oficio de la Inquisición, así como la de Obispo Auxiliar de Lima y de Huamanga. Sus estudios los realizó en la Universidad de San Marcos donde obtuvo el grado de doctor en Teología, desempeñándose luego como catedrático de Artes de Nona, de Prima de Sagrada Escritura y de Prima de Teología Escolástica. Falleció en Huamanga en $1748^{19}$.

José de Castro, limeño que llegó a ser Provincial de su orden religiosa y que por sus méritos profesionales obtuvo la confianza del Santo Oficio, donde fue nombrado Calificador, asímismo la del Virrey José Antonio de Mendoza, Marqués de Villa García quien lo designó como su confesor. En San Marcos se desempeño como catedrático de Prima de Santo Tomás; y en el Colegio Máximo de-San Pablo Tlego a ser Prefecto de Estudios Mayores $^{20}$. En este retrato el artista anónimo trabaja con sobriedad las vestiduras y centra nuestra atención en el rostro donde ha logrado una gran fuerza expresiva. En el fondo de biblioteca es posible ver una pintura de Santo Tomás de Aquino, la fuente de su cátedra sanmarquina.

\section{Biblioteca de Ietras}

Feliciano Palomares emercedariolèspañol nacido en 1679, pasó al Perú en 1719 donde llegó a ser Vicario General de su Orden en el Perú, Tierra Firme y Chile, asímismo Calificador del Santo Oficio. En 1725 por Real Cédula se creó en la Universidad de San Marcos la cátedra de Prima de Eximio Suárez, en la cual se enseñaba la doctrina del padre jesuita Francisco Suárez; el padre Palomares fue nombrado al año siguiente como el primer catedrático de esta disciplina instituida especialmente para la religión de La Merced. Murió en $1740^{21}$.

Entre los pintores más representativos de la escuela limeña del siglo XVIII se encuentran Cristóbal de Aguilar, José Joaquín Bermejo y Cristóbal Lozano. El Museo de Arte de San Marcos tiene el privilegio de contar en su colección con el retrato de don Pedro Peralta Barnuevo, firmado por

19 Eguiguren, L.A.: Op. cit., T. I, pp. 828-829.

20 Eguiguren, L.A.: Op. cit., T. I, pp. 854-855.

21 Eguiguren, L.A.: Op. cit., T. I, p. 827. Lima 1940. Pérez, Pedro Nolasco: Religiosos de La Merced. Segunda parte. Sevilla, 1923. 
Cristóbal de Aguilar y el retrato de don Pedro José Bravo de Lagunas, firmado por José Joaquín Bermejo. Hoy a la luz de la restauración nos permitimos atribuir el retrato del Martín de Andrés Pérez de la orden de San Camilo al pintor Cristóbal Lozano.

Cristóbal Lozano estuvo estrechamente ligado a la orden de San Camilo donde figuran lienzos no sólo de temática religiosa, sino también retratos de personajes de dicha orden hospitalaria, varios de ellos sin firma, pero que sin embargo figuran en las cuentas de los archivos del convento como encargados a Lozano. Entre dichas cuentas consta un pago hecho al pintor en 1771 por el retrato póstumo del Padre Pérez, fallecido en $1770^{22}$.

Creemos que por los caracteres estilísticos, por las referencias del archivo conventual, así como por la estrecha amistad entre Lozano y el Padre Pérez, quien incluso le dio diploma de hermano agregado a la Orden de San Camilo, es indudable que este lienzo fuera pintado por él. Fallecido Lozano fue enterrado en 1776 en la cripta de la iglesia de los camilos.

Martín de Andrés Pérez, fue por muchos años Lector de Teología y Maestro en el Colegio de Alcalá en España. Llegado a Lima se dedicó a la formación de los novicios de su orden, consiguiendo excelentes frutos, lo que le valió que el rector dela Universidad de-SanMarcos le ofreciera la cátedra de Prima de M̄ora, ecreađa èspecialmentepara su orden religiosa en 1754. Murió con fama de santidad en $1770^{23}$.

Los retratos de personajes de los siglos XIX y XX

Se ha incluido en la selección de retratos restaurados tres obras que corresponden a personajes que vivieron entre las últimas décadas del siglo XIX y la primera mitad del presente siglo; nos referimos a los retratos de Pedro Carlos Olaechea, Horacio Urteaga y Manuel Vicente Villarán.

El retrato de Pedro Carlos Olaechea está firmado por el pintor peruano Luis Astete y Concha, quien realizó estudios en España y fue posterior-

22 Mendiburu, Manuel de: Op. cit., t. VIII, pp. 320-321. Lima, 1934.

23 Eguiguren, L.A.: Diccionario Histórico..., t. I, p. 553. Lima, 1940; fd., Catúlogo Histórico..., p. 88. Lima, 1912; Vargas Ugarte, Rubén: Ensayo de un diccionario..., pp. 420-422. Burgos, 1968. 
mente profesor de dibujo en la Academia Concha en Lima. Astete destacó en el género retratístico, dentro de la línea de corte académico de fines del siglo XIX, como lo prueba este lienzo y otros que se conservan en el Museo de Arte de la Universidad.

Pedro Carlos Olaechea, nacido en Ica en 1865, realizó sus estudios en la Universidad de San Marcos donde se graduó de Doctor en Jurisprudencia en 1901 y posteriormente tuvo a su cargo la cátedra de Derecho Civil. Perteneció al Partido Demócrata y llegó a ser Diputado por Ica y Castrovirreyna. Murió en Rio de Janeiro en 1907.

El retrato del rector Manuel Vicente Villarán se encuentra firmado por Luis S. Ugarte en 1926. Pintor activo en Lima en las primeras décadas del presente siglo, quien fuera director de la Academia Concha y fundador de la Sociedad de Bellas Artes.

Manuel Vicente Villarán, limeno, nacido en 1876, realizó sus estudios en la Universidad de San Marcos donde se recibió de Doctor en Leyes en 1908. Incorporado a la docencia universitaria tuvo a su cargo las cátedras de Derecho Natural, Constitucional y Filosofía del Derecho, entre otras. En 1918 fue nombrado Decano de la Facultad de Jurisprudencia y en 1922 Rector de la Universidad. DefensoedelaFacuttadaleArtes de San Marcos. En 1936 fue candidato ada Presidendiả đe la/República y tres años más tarde Embajador en la Santa Sede. Murió en 1958.

Por último el retrato de Horacio Urteaga, obra anónima de similares características a las últimas citadas, retratos en busto de fondos neutros y pincelada académica.

Horacio Urteaga, nacido en Cajamarca en 1877, realizó sus estudios en la Universidad de San Marcos donde se graduó de Doctor en Jurisprudencia en 1904. Después de desempeñarse en diversas cátedras de su profesión, fue elegido en dos oportunidades Decano de la Facultad de Letras, en 1930 y 1935 . En su extensa currícula se cuentan innumerables cargos en instituciones culturales, entre los que podemos destacar: Director del Archivo Nacional, Director del Museo "Víctor Larco Herrera". Igualmente son innumerables sus publicaciones sobre historia del Perú. Murió en Lima en 1952. 
Este primer logro en actividades de restauración del patrimonio sanmarquino, es un ejemplo de lo que se puede conseguir cuando se aunan fuerzas y voluntades. Por ello para concluir estas líneas deseamos destacar la participación de los profesionales en restauración que pertenecen al equipo del Convenio Perú-España, así como a los alumnos de la Escuela Académico Profesional de Arte de la Facultad de Letras de San Marcos, que colaboran incondicionalmente en el logro de nuestros objetivos, de manera especial a la señorita Támira Basallo, asistente de la dirección y al señor Manuel Munive por su apoyo en las investigaciones.

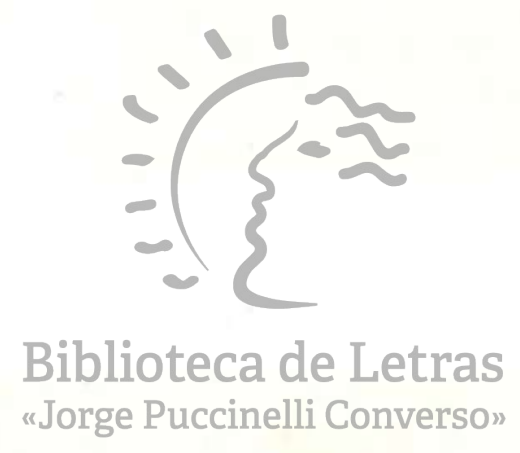




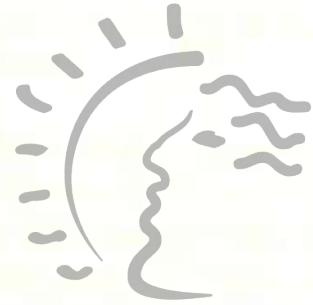

Biblioteca de Letras "Jorge Puccinelli Converso" 

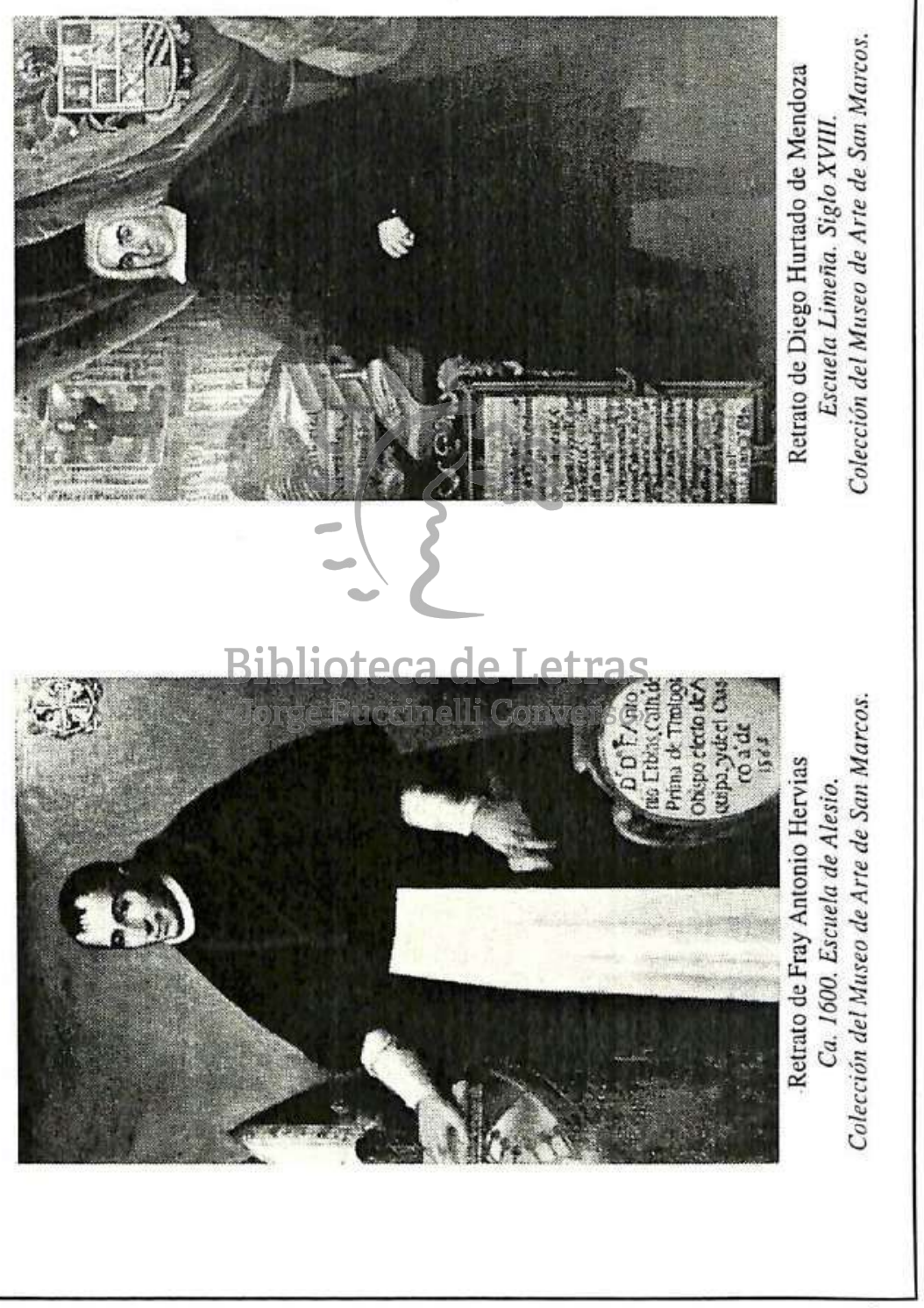


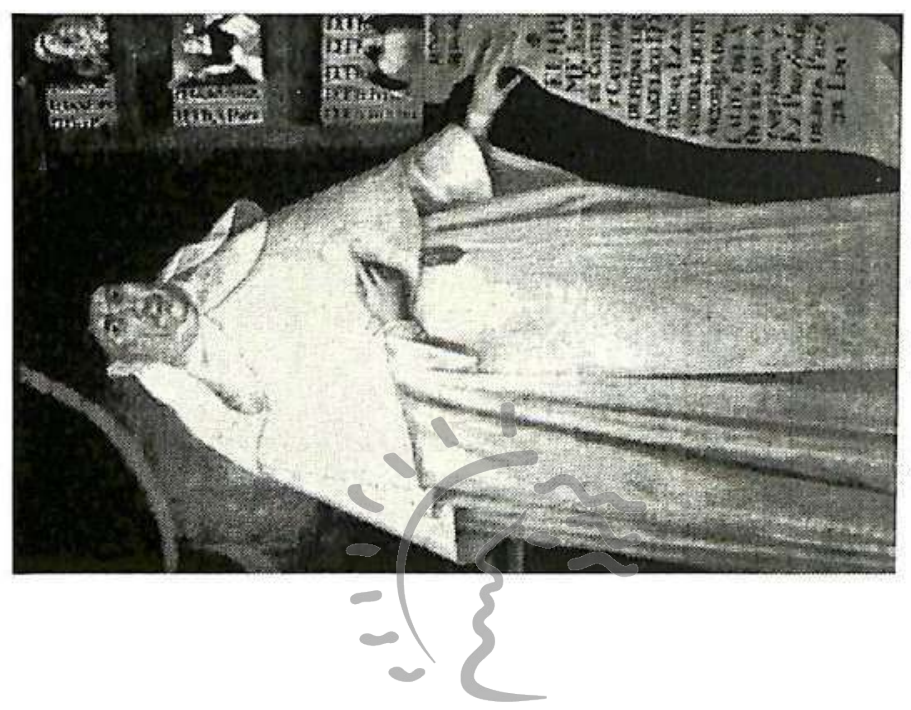

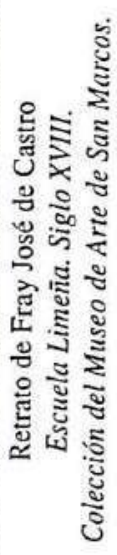
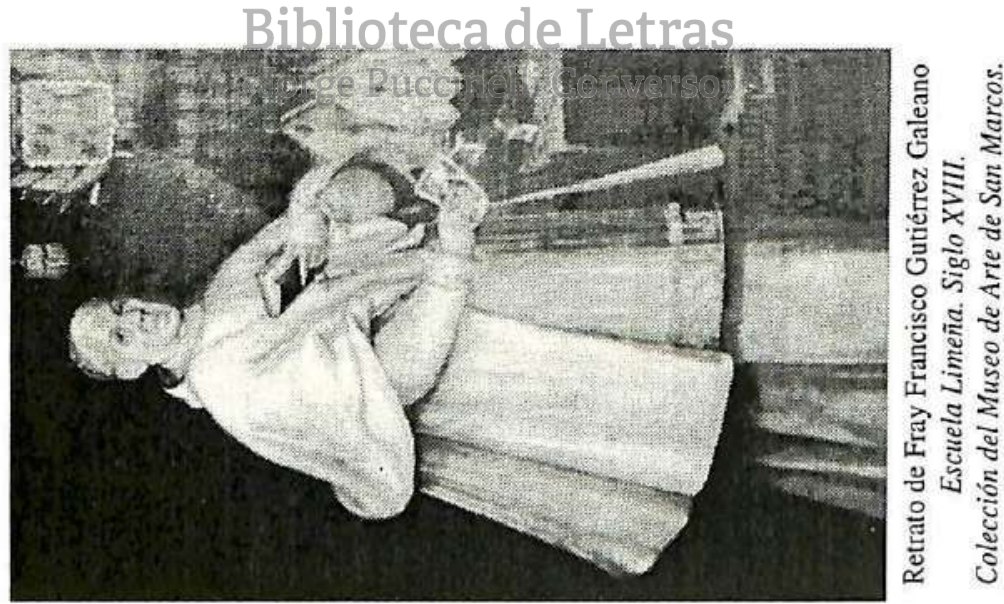


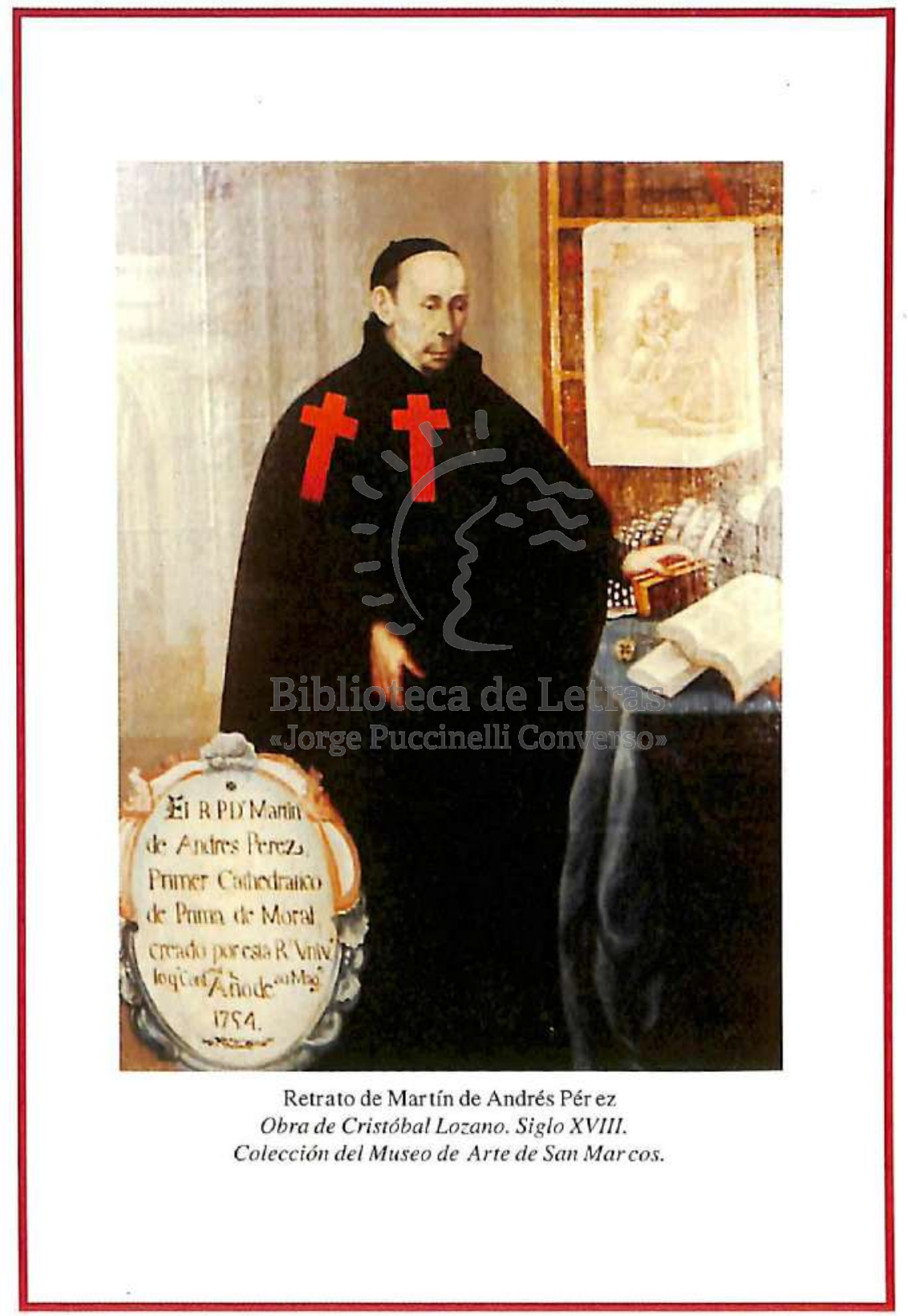




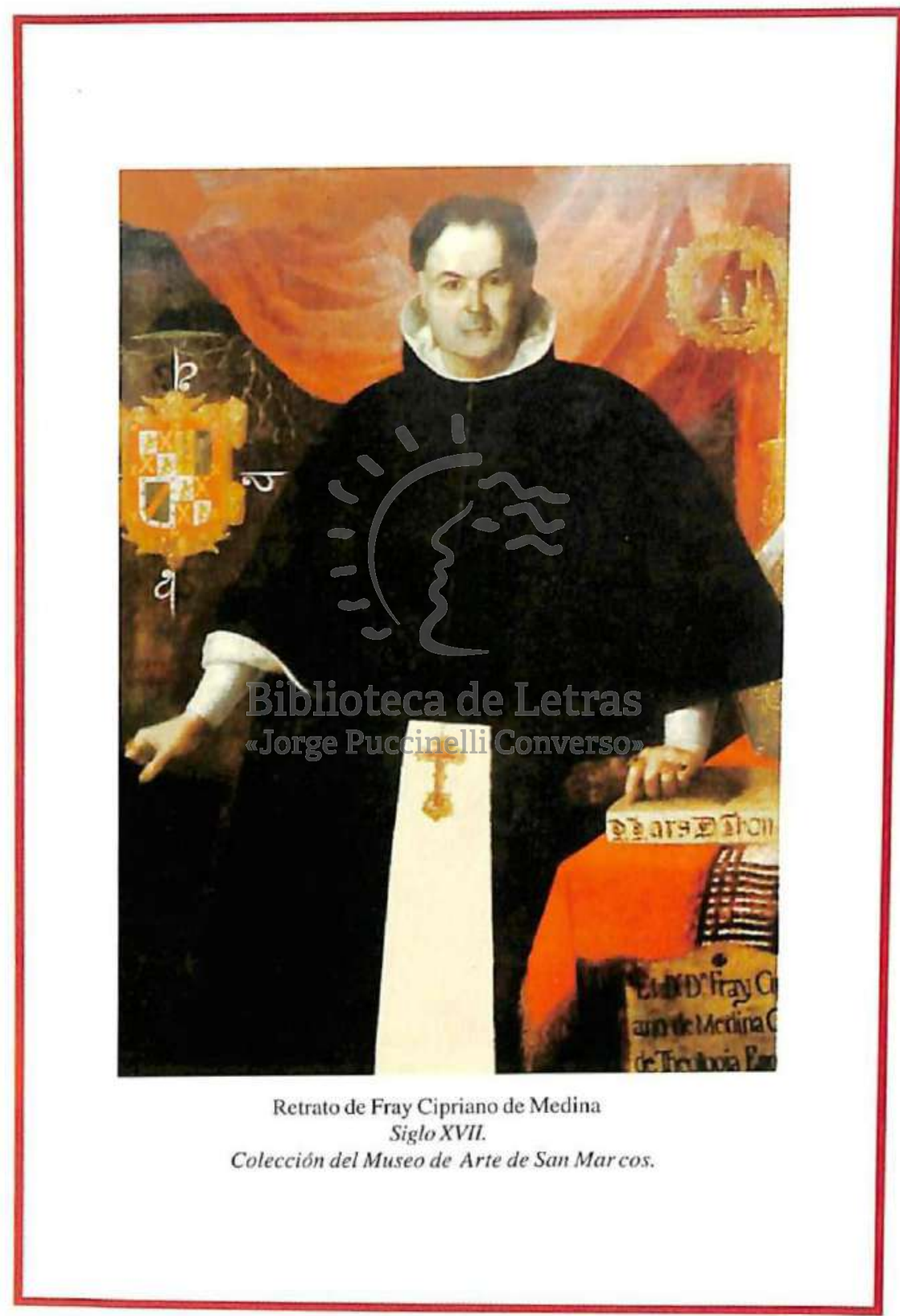




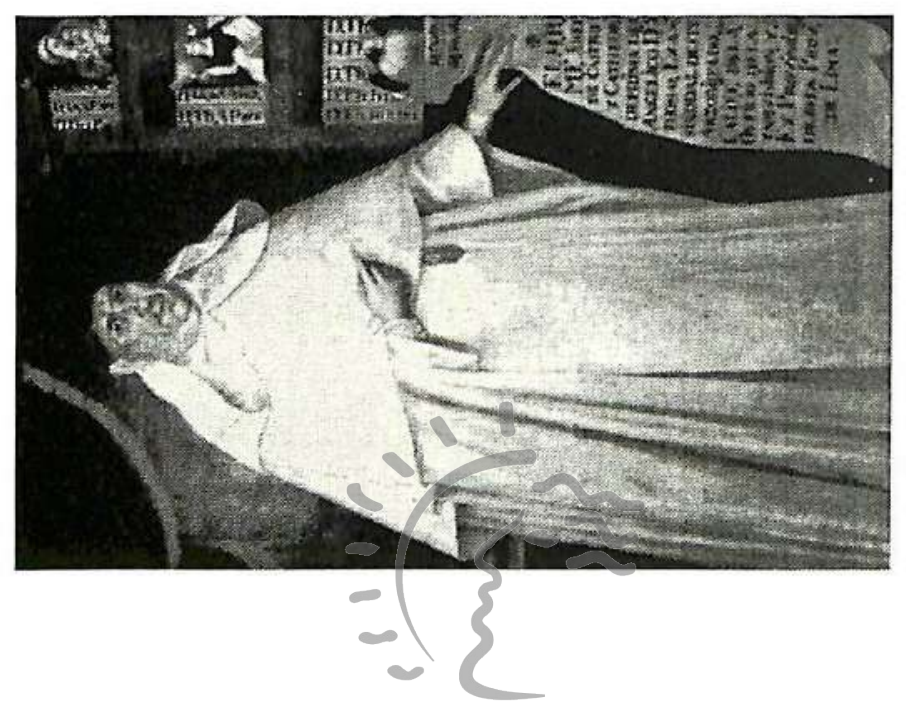

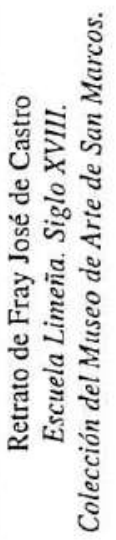
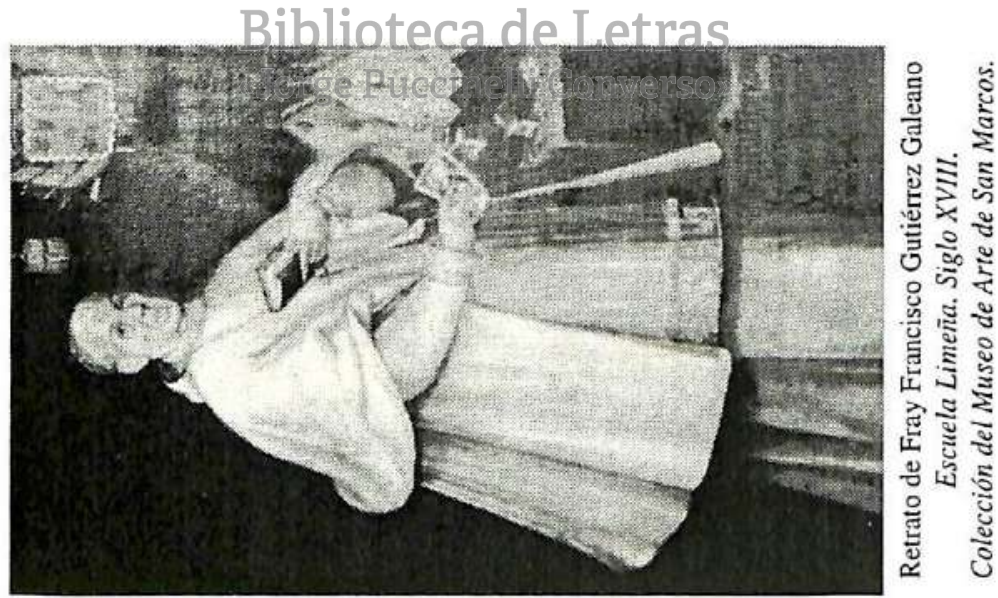